\title{
Quantitative Phenomics of Growth and Size in Animals
}

\author{
V. L. Stass ${ }^{1}$ \\ ${ }^{1}$ Laboratory of Animal Genetics, Institute of Animal Husbandry and Veterinary Sciences, Latvia \\ Correspondence: V. L. Stass, Laboratory of Animal Genetics, Institute of Animal Husbandry and Veterinary \\ Sciences, Latvia. E-mail: valbet.axon@apollo.lv
}

Received: April 1, 2019

doi:10.5539/ijb.v11n3p16
Accepted: April 25, $2019 \quad$ Online Published: May 4, 2019

URL: https://doi.org/10.5539/ijb.v11n3p16

\begin{abstract}
This study introduces a model of the growth phenotype dynamic in pigs by applying analytical methods. The model describes a transformation of the growth phenotype from $30 \mathrm{~kg}$ live weight up to the species maximum weight.

This paper focuses on a description of the determinants, which channel growth dynamic through animals' ontogeny. Theoretical notions about the growth are explicitly included in the concept. In the model, functional relations between relevant traits obtained in the experiments and field observations are analysed. Two focal variables, a feed conversion coefficient, and an invariant of growth are explicitly integrated in the model.

The novelty of the research is a proposition that deterministic, analytical model is a conceivable approach to studying a phenotype of a quantitative trait. According to this proposition, processes that contribute to the growth and development continue in later life till a species maximum weight is attained. The concept is formulated as a deterministic, hybrid model. In the model, both standard continuum methods and discrete-time difference equations have been used.

Applied to experimental data, the model has produced a new insight into the problem. In domestic pigs, the following three sets of ontogenetic growth phenotypes have been identified: one set with three species maximum weight phenotypes, one set with three rapid growth phenotypes, and one set with individual maximum weight phenotypes. In the study, not only the sets of growth phenotype are identified but also a possible interpretation of a conversion mode of the sets in ontogeny as well as a reading of growth trajectory dynamic are discussed.
\end{abstract}

Keywords: Ontogenetic Growth, Growth Phenotype, Quantitative Trait, Maximum Weight, Weight Invariant, Feed Conversion, Hybrid Model

\section{Introduction}

The purpose of this study was to introduce a concept of the growth phenotype dynamic in pigs' ontogeny. As soon as the growth rate phenotypes are identified, methods of the qualitative traits genetics become applicable to the growth analyses. The method applied in the study is mathematical modelling. To meet the study purpose, two new relevant variables are introduced. Parameter ' $\mathrm{K}$ ', an invariant of growth, and a feed conversion coefficient, ' $\mathrm{Z}$ ' are focal variables in the model. Along with the new variables, a deterministic hybrid model of growth was built. A biological interpretation of the results in terms of quantitative traits genetics brings a theoretical aspect in the study.

\subsection{Phenomics of Quantitative Traits}

Phenomics, the comprehensive study of phenotypes, is essential to understanding biology (Houle, 2010). Phenotypes arise from principally nonlinear biological processes underlying developmental, signalling, and metabolic cascades (Marjoram et al., 2014). Despite an extensive amount of accumulated experimental data, our understanding of the biological processes that direct growth is far from complete. On the other hand, our limited ability to understand many important biological phenomena suggests that we are not measuring or have not identified all relevant variables (Houle et al., 2010).

In this study, we use the opinion that in multi-cellular organisms, phenotypic variation is variation in developmental trajectories throughout the ontogeny (Fusco \& Minelli, 2010). Growth is not an isolated phenomenon, rather it should be viewed as a dynamic system in which multiple biological aspects are coordinated to control growth processes ( $\mathrm{Li} \& \mathrm{Wu}, 2010)$. Body size is one of the most fundamental attributes of any organism. 
This statement is supported by the observation that body size influences nearly every aspect of the biology of organisms and that many life history variables correlate with body size (Werner \& Griebeler, 2011). The regulation of body size in animals ontogeny remains a problem in developmental biology. The size to which an individual animal grows is affected by both genetic and environmental factors. Current level of understanding of many features of quantitative traits is quite rudimentary; traits such as size or longevity vary greatly among individuals and have continuously distributed phenotypes that do not show simple Mendelian inheritance (Hill, 2010). The central challenge of modern genetic analysis is to understand the biological determinants of quantitative phenotypic variation (Andersson \& Georges, 2004; Trudy et al., 2009; Cobb et al., 2013). Phenotypic selection has created a wide variety of breeds of domestic animals. Domestic animals therefore constitute a unique resource for understanding the genetic basis of phenotypic variation (Andersson \& Georges, 2004). It is recognised that variation in the growth rate of pigs starts from conception, with pigs of the same litter often varying considerably in birth weight. This variation in pig growth performance both within and between litters continues through their lifetime (Magowan et al., 2007). We know very little about how the distribution of theoretically possible phenotypes originates through the process of ontogeny. What is missing is a theory, or at least a more systematic, quantitative, and integrative understanding of ontogeny, including metabolism, physiology, and development (Jaeger \& Monk, 2014).

\subsection{Growth Phenotype}

The following considerations serve as a theoretical base for the concept. Growth phenotype varies through animal lifetime. Animal size and growth rate change during ontogeny and are contingent upon both genetic and environmental factors. Moreover, phenotypes vary from cell to cell and from moment to moment (Houle et al., 2010). How to distinct or identify growth phenotypes taking into consideration the above theoretical standpoints? If growth phenotype changes during animal ontogeny model as a dynamic system with a few causal variables then there are some possibilities to identify growth phenotype in individual animals. The first option to identify the phenotypes is at the growth point where individual animal weight reaches maximum, the second option is at the growth point where a species maximum weight is attained, and third option is at the growth rate maxima points during rapid growth. At these body weight or growth rate maxima points animals' growth stops, or temporarily stops. As a result, growth or body weight phenotypes can be explicitly identified due to the fact that the phenotypes, which reached their asymptotic weight or maximum growth rate points cannot vary in the points. At these maxima points, the trait's determinants cannot alter phenotype. To test these theoretical notions, a deterministic model of growth phenotype dynamic was built.

\section{Materials and Methods}

The dynamic of growth phenotype in domestic pigs has been modelled and discussed in terms of body weight, daily gain, and feed conversion coefficient. Two focal variables have been used in the model, an invariant of growth, $\mathrm{K}$, and a feed conversion coefficient, $\mathrm{Z}$. The variables are not conventional biological traits, cannot be directly measured in animals, and are dimensionless.

\subsection{Data Set}

The data set was obtained from experiments on growing domestic pigs, fed from $30 \pm 3 \mathrm{~kg}$ up to $96 \pm 5 \mathrm{~kg}$ live weight. The animals had been chosen for the experiments at random and proportionally from crosses between four breeds. The pigs were housed and fed under non-industrial conditions, either in a pig testing station or in research facilities. The experiments were performed in accordance with Declaration of Helsinki, Livestock regulations, National legislation, and institutional rules.

\subsection{The Model's Variables}

' $\mathrm{M}$ ' stands for an individual animal current live weight, measured in kilograms.

$M=\left\{M \in \mathbb{R}_{+} \mid 30 \leq M \leq 600\right\}$, an animal individual maximum weight, $M_{x}=600 \mathrm{~kg}$.

' $\mathrm{m}$ ' is an animal initial considered weight, measured in kilograms, $\mathrm{M} \geq \mathrm{m}, \mathrm{m}_{\mathrm{o}}=30 \mathrm{~kg}$.

' $\mathrm{t}$ ' is chronological discrete current time, measured in days from animals' birth.

$\mathrm{t}=\{\mathrm{t} \in \mathbb{N} \mid 0<\mathrm{t}<\infty\}, \Delta \mathrm{t}=1,2,3 \ldots \mathrm{n}, \mathrm{n} \in \mathbb{N}$.

' $\mathrm{t}_{\mathrm{o}}$ ' is starting time, related to $\mathrm{m}_{\mathrm{o}}, \mathrm{t}_{\mathrm{o}}=90$ days.

' $\mathrm{K}$ ' is an invariant of growth, considered as a parameter, dimensionless.

$\mathrm{K}=\left\{\mathrm{K} \in \mathbb{R}_{+} \mid 1 \leq \mathrm{K}<11\right\}, \mathrm{K}_{\mathrm{o}}=1$.

' $\mathrm{Z}$ ' is a current feed conversion coefficient, dimensionless. 
$\mathrm{Z}=\left\{\mathrm{Z} \in \mathbb{R}_{+} \mid \mathrm{Z}_{\mathrm{o}} \leq \mathrm{Z}<\infty\right\},(\mathrm{Z}=\infty) \rightarrow\left(\mathrm{M}=\mathrm{M}_{\max }\right), \mathrm{Z}_{\mathrm{o}}$ is related to $\mathrm{m}_{\mathrm{o}}$.

\subsection{Mathematical Method}

In this study, a hybrid modelling technique (Aihara \& Suzuki, 2010) was applied. The benefit of using the hybrid analytical discrete-continuous model is that it helps integrate the knowledge about the pig biology and performance. Such models can produce complicated dynamics, coexistence of multiple complicated attractors, cycles, chaos, even in low dimensions (Mailleret \& Lemesle, 2009). Hybrid dynamical systems combine evolution equations with state transitions. When the evolution equations are discrete-time, also called map-based, the result is a hybrid discrete-time system (Cao \& Ibarz, 2010).

In the model, discrete-time and continuum modelling techniques have been combined to form a dynamic system. Growth phenotype dynamic in pigs kept under nonindustrial conditions from $30 \mathrm{~kg}$ up to species maximum weight was modelled.

\section{Results}

Rapid growth in domestic pigs has been observed between $30 \mathrm{~kg}$ and $96 \mathrm{~kg}$ live weight. In this weight range, growth rate maxima have been reported in most pigs. The aim of this section is to identify growth phenotypes. I shall remind the interested reader some theoretical details needed to follow the model. Physiologically, growth rate in domestic pigs has maxima. The growth rate maxima are genetically determined. Identification of animal growth phenotypes during the rapid growth is associated with finding the growth rate maxima. When a maximum of growth rate is reached, an animal's live weight does not change for a while. Right under these conditions it is possible identify growth phenotype. A growing pig unavoidably has individual growth rate maximum, which characterises both its growth ontogenetic trajectory and growth phenotype. It is clear, growth phenotypes must follow the Mendel rules, consequently the concept is experimentally testable. The task is to find out a set with rapid growth rate maxima and give it genetic interpretation.

\subsection{Preliminary Mathematical Results}

Parameter $\mathrm{K}$ is an invariant of growth or weight, it is equal for the same weight pigs $\left(\mathrm{M} \geq \mathrm{m}_{\mathrm{o}}\right)$ regardless of their daily gain. Parameter K has the following form:

$$
K=\frac{M t}{m_{o}\left(2 t-t_{o}\right)}
$$

The system (2) comprises main experimental facts and serves as the model's base, given by

$$
\left\{\begin{array}{l}
\frac{M}{m_{o}}=2 K-1+\frac{\left(t-K t_{o}\right)}{t} \\
\frac{\left(t-K t_{o}\right)}{t}=\frac{(Z-2 K) \cdot(K-1)}{Z K}
\end{array}\right.
$$

From system (2) follows

$$
\frac{1}{m_{o}} \cdot \frac{\Delta M}{\Delta K}=\frac{2 K-1}{K}-\frac{2}{Z}
$$

Data analyses acquired from pig-breeding farms where mature boars were kept proved that typical maximum individual weight was $M=M_{x}=600 \mathrm{~kg}$. Expression (4) converges as $M \rightarrow M_{x}$, and the following limit holds:

$$
\lim _{M \rightarrow M_{x}}\left(\frac{2 K-1}{K+1}\right)=\sqrt{3}, \text { where }\left.\left(K \rightarrow K_{x}\right)\right|_{M \rightarrow M_{x}}
$$

It follows from the limit that under condition $K=K_{x}, K_{x}=5+3 \sqrt{3}=10,19615$. From (2) and (4) one can derive the following equations

$$
\begin{gathered}
Z_{x}=\frac{2 K_{x}\left(K_{x}-1\right)}{3},\left.Z\right|_{M=M_{x}}=Z_{x} \\
\frac{M_{x}}{m_{o}}=2 K_{x}-\frac{4}{K_{x}} \\
\frac{1}{m_{o}} \cdot \frac{\Delta M_{x}}{\Delta K_{x}}=\frac{\left(M_{x} \cdot K_{x}+m_{o}\right)+m_{o}\left(4 K_{x}^{3}-10 K_{x}-5\right)}{m_{o}\left(4 K_{x}^{3}-5 K_{x}\right)-K_{x}\left(M_{x} \cdot K_{x}+m_{o}\right)}
\end{gathered}
$$




\subsection{Rapid Growth Phenotypes}

Consider equation (3). This equation describes growth rate, and setting it to zero, one would obtain condition for the local maximum to exist. Substituting (5) into (3) and setting the result to zero one obtains $K=1,686$. This is the reason to consider a local growth rate maximum at $\mathrm{K}=1,686$. Consider equation (7), substituting (6) into (7) we have

$$
\frac{1}{m_{o}} \cdot \frac{\Delta M}{\Delta K}=\frac{4 K^{3}+2 K^{2}-10 K-5}{2 K^{3}-5 K}
$$

Considering this equation as a dynamic system, condition under which a steady state point exist is the following

$$
\frac{4 K^{3}+2 K^{2}-10 K-5=0}{2 K^{3}-5 K=0}
$$

it follows, $K=\sqrt{ } 2,5=1,581$. This is therefore an unstable steady state point. This suggests that at $K=1,581$ a local growth rate maximum is reached. The growth rate maximum found at $\mathrm{K}=1,686$ differs from this case. The finding suggests that there are not two growth rate local maxima, rather there is single local maximum of each growth phenotype. The results suggest that both rapid growth and species maximum weight growth trajectories are phenotype-dependant.

In the section above, two rapid growth phenotypes were identified at the following points $\mathrm{K}=1,686$ and $\mathrm{K}=1,581$ applying discrete-time difference equation technique. Below, to identify one more rapid growth phenotype, continuum variables technique is used. If in the section above the maximum growth rate points were found out directly, by analysing growth rate, then in this section, a different approach is applied. This approach is based on the fact that the closer to unity feed conversion coefficient, $\mathrm{Z}$ the higher growth rate. The general scheme is as follows. In the first step it is necessary find out a phenotype, $M_{a}$ that cannot reach $M_{x}=600 \mathrm{~kg}$. After that it is necessary find out a corresponding feed conversion coefficient, $Z_{a}$ and then find its minimum. $\mathrm{M}_{a}$ was found as integral average considering $\mathrm{M}_{x}=600 \mathrm{~kg}$. However, at first, I am going to find $\mathrm{K}_{a}$ that correspond $\mathrm{M}_{a}$. In this section, $\mathrm{K}_{x}$ and $\mathrm{Z}_{\mathrm{x}}$ have been considered variables. Integral average of parameter $\mathrm{K}$, we denote it $\mathrm{K}_{a}$, considering $\mathrm{K}_{\mathrm{x}}$, given by

$$
K_{a}=\frac{1}{Z_{x}} \cdot \int K_{x} d Z \quad, \quad Z_{x}=\frac{2 K_{x}\left(K_{x}-1\right)}{3}
$$

From (8) follows analytical expression of $\mathrm{K}_{a}$ given by

$$
\mathrm{K}_{a}=\frac{\mathrm{K}_{\mathrm{x}}^{2}\left(4 \mathrm{~K}_{\mathrm{x}}-3\right)}{9 \mathrm{Z}_{\mathrm{x}}}
$$

Numerically, $\mathrm{K}_{a}=6,9822$. The corresponding $\mathrm{M}_{a}$ given by

$$
\frac{M_{a}}{m_{o}}=2 K_{a}-\frac{4 K_{a}}{K_{x}^{2}}
$$

Numerically, $\mathrm{M}_{a}=410,874 \mathrm{~kg}$. The corresponding feed conversion coefficient, $\mathrm{Z}_{\mathrm{M} a}=95,388$. An analytical form of $Z_{\mathrm{M} a}$ is

$$
Z_{\mathrm{M} a}=2 K_{a}^{2}-f\left(K_{a}, c\right)
$$

Integral average, $\mathrm{Z}_{a}$ considering $\mathrm{Z}_{\mathrm{M} a}$ has the following form

$$
Z_{a}=\frac{1}{K_{a}} \cdot \int Z_{\mathrm{M} \alpha} d K
$$

It follows from (12) that analytical expression of $Z_{a}$ is

$$
Z_{a}=\frac{2 K_{a}^{2}}{3}-\sqrt{3} \cdot \operatorname{arctg}\left(K_{a}-1\right)
$$

There is a standard procedure how to find minimum $Z_{a}$. Minimum $Z_{a}$ was found when solving the following equation 


$$
\frac{d z_{a}}{d K_{a}}=0
$$

Solution to the equation (14) is $\mathrm{K}_{a}=1,232$. This value of parameter $\mathrm{K}$ minimises $\mathrm{Z}_{a}$, and this is the reason that $\mathrm{K}=$ 1,232 is considered as a point where a local maximum of rapid growth rate is attained.

\subsection{Species Maximum Weight Phenotypes}

In this section, a set with species maximum weights has been found. The species maximum weight can be attained by the animals that have passed through bifurcation point at $\mathrm{M}_{\mathrm{x}}$. Only some animals at the attainment of the $M_{x}$ can pass through the bifurcation point and take on the growth trajectory $\left(M_{x} \rightarrow M_{x x}\right)$ that leads to the set with the species maximum weight phenotypes.

The following two equations (15), (16) were used to find out a set with species maximum weights.

$$
\begin{gathered}
K_{x}^{2}-\frac{K_{x} \cdot M_{x}}{2 m_{o}}-2=0 \\
\frac{1}{m_{o}} \cdot \frac{\Delta M_{x}}{\Delta K_{x}}=\frac{\left(M_{x} \cdot K_{\chi}+m_{o}\right)+m_{o}\left(4 K_{x}^{3}-10 K_{x}-5\right)}{m_{o}\left(4 K_{x}^{3}-5 K_{x}\right)-K_{x}\left(M_{x} \cdot K_{x}+m_{o}\right)}
\end{gathered}
$$

The equations one can derive from system (2), (3) and (5), (6). I can remind the interested reader that $\mathrm{K}_{\mathrm{x}}=$ $\left.\mathrm{K}\right|_{\mathrm{M}=\mathrm{Mx}}$. To find out the species maximum weights we must find maximum $\mathrm{M}_{\mathrm{x}}$ in equations (15) and (16). There is a standard procedure how to find maximum $M$ in equation (15). Since equation (15) is convex, sufficient condition for the maximum $M$ to exist is fulfilled. Maximum $M_{x x}$, we denote it $M_{x x 1}$, analytical expression given by

$$
M_{x x 1}=4 K_{x} m_{o}
$$

Considering equation (16) one can notice that maximum $M_{x x}$ in (16), we denote it $M_{x x 3}$, is expected in the following form

$$
M_{x x 3}=\frac{2 m_{o}\left(2 K_{x}^{2}-3\right)}{K_{x}}
$$

Average of (17) and (18), we denote it $\mathrm{M}_{\mathrm{xx} 2}$, given by

$$
M_{x x 2}=\frac{m_{o}\left(4 K_{x}^{2}-3\right)}{K_{x}}
$$

The three species maximum weights, (17), (18) and (19) forms set A with the species maximum weight phenotypes.

In the section below has been verified that the above results (17), (18) and (19) are not just species maximum weight estimations, they are phenotypes. To prove this statement, two initial results are needed. The first follows from (19)

$$
\frac{1}{m_{o}} \cdot \frac{\Delta M_{x x 2}}{\Delta K_{x}}=\frac{4 K_{x}+3}{K_{x}}
$$

In the following analyses, $M_{x}, K_{x}, t_{x}, Z_{x}$, are regarded as variables. Consider (18) and (5), each of the equations was obtained in an independent procedure. The result is given by

$$
\frac{1}{m_{o}} \cdot \frac{\Delta M}{\Delta K}=\frac{3 Z(K+1)-2 K+K Z}{K Z}
$$

The limit $(Z \rightarrow \infty)$ of $(21)$ is exactly $(20)$. Passion to the limit procedure reveals an internal connection between (18) and (19). The procedure is certainly correct; it arises from the following reason: $\left(M \rightarrow M_{x x}\right) \Rightarrow(Z \rightarrow \infty)$. In the next stage, more relations between (17), (18) and (19) are found. Consider a system of (17) and (20). I combine two results, obtained by different methods, considering them related, given by

$$
\frac{1}{m_{o}} \cdot \frac{\Delta M}{\Delta K}=4+\frac{3}{K}=\frac{M}{K \cdot m_{o}}+\frac{3}{K}=\frac{4 K^{2}+3 K-3}{K^{2}}
$$


Equation (22) is biased; it is not in full agreement with (20) due to a wrong constant -3 . The task is to find the correct constant. I write (22) in a different form:

$$
\frac{1}{m_{o}} \cdot \frac{\Delta M}{\Delta K}=\frac{4 K^{2}+3 K-X}{K^{2}}
$$

where $X$, a constant, is unknown. In the following, $X$ will be found. Considering (20) and (23), it follows: $(X \rightarrow 0) \Leftrightarrow(Z \rightarrow \infty)$. It is a general statement; however, in this case, under the current approach, $\left.Z\right|_{(M \rightarrow M x x)}=Z_{x x} \neq \infty$, and $X$ is a constant. It means $(X \rightarrow$ constant $)$ and $\left(Z \rightarrow Z_{x x}\right)$. Further analysis leads to the following results: $2 K / X$ $=K_{x x} \cdot Z_{x}$, where $Z_{x x}=K_{x x} \cdot Z_{x}$. It follows $\left(M_{x} \rightarrow M_{x x}\right) \Rightarrow\left(Z_{x} \rightarrow Z_{x x}\right)$. As a result, corresponding $K, K_{x x}$ was found, given by

$$
K_{x x}=K_{x}-\frac{1}{K_{x}}
$$

The effort put into finding constant $X$, has led to identification of two constants, $K_{x x}$, and $Z_{x x}$, associated with $\mathrm{M}_{\mathrm{xx}}$. Taking into consideration the new constants, $\mathrm{K}_{\mathrm{xx}}$, and $Z_{\mathrm{xx}}$, the species maximum weight growth phenotypes given by

$$
\begin{aligned}
& \frac{1}{m_{o}} \cdot \frac{\Delta M_{x x 1 \backslash x}}{\Delta K_{x x}}=\frac{2\left(K_{x}^{2}+2\right)}{K_{x}\left(K_{x}-1\right)-1} \\
& \frac{1}{m_{o}} \cdot \frac{\Delta M_{x x 2 \backslash x}}{\Delta K_{x x}}=\frac{2 K_{x}^{2}+1}{K_{x}\left(K_{x}-1\right)-1} \\
& \frac{1}{m_{o}} \cdot \frac{\Delta M_{x x 3 \backslash x}}{\Delta K_{x x}}=\frac{2\left(K_{x}^{2}-1\right)}{K_{x}\left(K_{x}-1\right)-1}
\end{aligned}
$$

We denoted $\Delta \mathrm{M}_{\mathrm{xxn} \mid \mathrm{x}}=\mathrm{M}_{\mathrm{xxn}}-\mathrm{M}_{\mathrm{x}}$, where $\mathrm{n}=1,2,3$. And $\Delta \mathrm{K}_{\mathrm{xx}}=\mathrm{K}_{\mathrm{xx}}-1$.

The same set of variables has been used to model animals' growth, and growth phenotypes: $M$, animals current weight; $t$, current discrete time; $K$, an invariant; $Z$, a feed conversion coefficient. Subscript $x$, for example $M_{x}$, denotes variable at a certain point. For example, animals individual maximum weight, $M_{x}=600 \mathrm{~kg}$, accordingly $K_{x}, Z_{x}$ and $t_{x}$ correspond to $M_{x}$. $M_{x x}$ denotes a species maximum weight, and accordingly $K_{x x}, Z_{x x}$ and $t_{x x}$ correspond to $\mathrm{M}_{\mathrm{xx}}$. It follows from the model that species maximum weight phenotype forms set $\mathrm{A}$; the set is possible describe in the following form:

$$
\begin{array}{llr}
\text { AA } & \text { Aa } & \text { aa } \\
\left(\mathrm{M}_{\mathrm{xx} 1}\right. & \mathrm{M}_{\mathrm{xx} 2} & \left.\mathrm{M}_{\mathrm{xx} 3}\right) \vee \\
\left(\frac{\Delta M_{x x 1 \backslash x}}{\Delta K_{x x}}\right. & \frac{\Delta M_{x x 2 \backslash x}}{\Delta K_{x x}} & \left.\frac{\Delta M_{x x 3 \backslash x}}{\Delta K_{x x}}\right)
\end{array}
$$

It follows that a feasible interpretation is that a single two allelic gene, A, determines species maximum weight. A most unusual feature is that to find out the phenotypes, a combination of discrete-time and continuum methods was needed. Neither of the methods separately could produce the result.

\section{Discussion}

Our results support the opinion that modelling of biological function has an essential role to play in unravelling genetic causation (Noble, 2008). The power of mathematical modelling lies in its ability to reveal previously unknown or counterintuitive principles that might have been overlooked or missed by a qualitative approach to biology (Altrock et al., 2015).

The insight that follows concern a possible interpretation of the results. Do quantitative trait phenotypes have an emergent nature or do they have an asymptotic nature? If the last question is answered in the affirmative, then, under the model conditions, the phenotypes are unstable most of the time and could only be identified asymptotically. That we can measure and calculate the weight in animals at any time does not mean this is an argument to the contrary. Unstable phenotypes can be measured and calculated, but the calculations usually do not agree with measurements, except at the asymptotic, extremum, or steady state points. This reasoning is 
supported by the opinion that phenotypes vary from cell to cell and from moment to moment and therefore can never be completely characterized (Houle et al., 2010).

However, with our present knowledge, is it possible to reconcile the assumption that a growing organism, for most of the time, is in an unstable dynamic process with a limited number of knot-points, can yield normal analyses? What does this supposed instability amount to? It is an unstable growth trajectory towards a stable state in the dynamic system's phase space. This unstable trajectory is a mathematical abstraction. Although interpretations of unstable trajectories are possible in the case of mechanical systems, it is problematic when applied to biological growth. The question is how can unstable processes result in a stable form and function? What is it that that brings order to unstable processes? Is it genetic information? Taking this position, it is feasible to speculate that the genetic information does not supervise the whole process of growth, but only some knot-points, through which growth trajectory must pass in a certain order when a phenotype is forming. The supposed knot-points are a limited number of sets, which specify growth trajectory and are therefore genetically determined. The complete number of the sets could be acquired from the analyses of extended model and is related to the growth phenotype trajectory.

From the above reasoning, it follows that there are a considerable number of growth trajectories at the rapid growth stage; each trajectory, however, passes through one of the three growth rate maximum points. It is important to note that although there are only three growth rate maximum points, each animal at each of these points have its own unique instantaneous growth rate. This means that animals with the same growth rate phenotype may have different, their unique growth rates at the maximum growth rate point. It follows that the individual maximum growth rates form a set of values for each phenotype and not a single value. This is understandable and explains growth rate dynamic in both domestic animals reared on a variety of feeds, and wild animals with different foraging abilities and, thereby, various body weights.

\subsection{Growth Phenotype Dynamic}

It is common opinion that growth curve in most animals is smooth, continuous, and sigmoid in form. This form of growth curve has been considered universal and was modelled by a number of growth functions. Most growth is sigmoid, with a slow and gradual start and an equally slow and gradual termination as the final size is approached (Nijhout \& German, 2012). The model shows this is not the case in the pig. Under the model conditions, growth curve is neither smooth nor continuous. There is a bifurcation of the growth trajectory and an inflection point after bifurcation. At these curve points, growth trajectory qualitatively changes.

A number of animal growth models apply implicit statement that in mammals, mass at maturity, average adult mass, and maximum mass are likely to be quite similar (Economo et al., 2005). The model shows this is not the case in the pig. Under the model conditions, in the course of ontogenetic growth in pigs it is possible distinct three sets of growth phenotype, whereas average weight, individual maximum weight, and species maximum weight differ significantly.

\subsection{Rapid Growth Phenotypes}

Rapid growth phenotypes form set $B$, with the following three maximum growth rate phenotypes: $\{B \mid K=1,686$; $\mathrm{K}=1,581 ; \mathrm{K}=1,232\}$ that correspond to $\{\mathrm{B} \mid \mathrm{BB} ; \mathrm{Bb} ; \mathrm{bb}$ \} respectively. Maximum at $\mathrm{K}=1,686$ have animals with live weight about $70 \mathrm{~kg}$, maximum at $\mathrm{K}=1,581$ have animals with live weight about $60 \mathrm{~kg}$, and maximum at $\mathrm{K}=1,232$ have animals with live weight about $45 \mathrm{~kg}$. There are three options for the growth trajectories to develop. One trajectory can be denoted $(\Delta \mathrm{Z}<0)$. At $\mathrm{m}_{\mathrm{o}}$, a phenotype, by a yet unknown process or a gene set to grow along one of three conceivable trajectories. It means that at the beginning of rapid growth trajectory $(\Delta \mathrm{Z}<0)$ the animals' current feed conversion coefficient become less than their feed conversion, $Z_{0}$. These phenotypes are able reach maximum growth rate at $\mathrm{K}=1,232$, or $\mathrm{K}=1,581$, and then take on the $(\Delta \mathrm{Z}>0)$ growth trajectory. On the other hand, second possible growth trajectory $(\Delta Z>0)$ is inaccessible for those phenotypes. Passage from $(\Delta Z<0)$ to $(\Delta Z>0)$ or vice versa, in the range $1<\mathrm{K}<1,581$ is probable for animals only under special conditions, if at all. Growth trajectory $(\Delta Z>0)$ has usually been observed. Growth trajectory $(\Delta Z=0)$ was not analysed. However, growth trajectory $(\Delta \mathrm{Z}<0)$ is complicated and at this stage only its phenomenological description is possible.

\subsection{Individual Maximum Weight Phenotypes}

At this stage, the whole set of individual maximum weight phenotypes is impossible identify explicitly. The set consists of the phenotypes, which reached or can reach their maximum individual weight, $\mathrm{M}_{\mathrm{x}}$, and phenotypes that cannot reach individual maximum weight. This set is denoted $\mathrm{U},\left\{\mathrm{U} \mid \mathrm{M}=\mathrm{M}_{\mathrm{x}} ; \mathrm{M} \leq \mathrm{M}_{\mathrm{x}} ; \mathrm{M}<\mathrm{M}_{\mathrm{x}}\right\}$. Identification of the phenotypes that cannot reach their individual maximum weight is the model's next task. 


\subsection{Growth Trajectory Bifurcation}

An opinion that rapid growth rate phenotypes, set $\mathrm{B}$, in the course of ontogenetic growth smoothly transform themselves into species maximum weight phenotypes, set A, is biased. One fact prevents from establishing a smooth and continuous ontogenetic succession between the two sets of the phenotype. This fact is bifurcation of growth trajectory at the point animals attain their individual maximum weight. At this point $\left(\mathrm{M}_{\mathrm{x}}, \mathrm{K}_{\mathrm{x}}, \mathrm{Z}_{\mathrm{x}}, \mathrm{t}_{\mathrm{x}}\right)$ bifurcation of the growth trajectory takes place with two new trajectories to develop. On one trajectory $\left(\mathrm{m}_{\mathrm{o}} \rightarrow \mathrm{M} \rightarrow \mathrm{M}_{\mathrm{x}} \rightarrow \mathrm{M}_{\mathrm{x}}\right)$ animals grow up until individual maximum weight is attained and later continue remain constant. On other growth trajectory $\left(\mathrm{M}_{\mathrm{x}} \rightarrow \mathrm{M}_{\mathrm{xx}}\right)$, after bifurcation, phenotypes continue to grow up to the species maximum weight is reached. Findings suggest that not all phenotypes are able to reach individual maximum weight. It follows from the model that only some phenotypes can attain bifurcation point, and only some of them go through the bifurcation and at this point take on the trajectory that leads to a species maximum weight phenotype. It means that in-between the two trajectories there is a gene or process, which either stops or enables certain growth mode. Considering genetic regulation, it is feasible to propose that abovementioned bifurcation is under genetic control and has been brought about by interaction of two genes, $\mathrm{U}^{\circ} \mathrm{G}$, where $\mathrm{G}$ is a gene modifier, and $\mathrm{U}$ is a gene of individual maximum weight.

Species maximum weight phenotype forms on the growth trajectory $\left(\mathrm{M}_{\mathrm{x}} \rightarrow \mathrm{M}_{\mathrm{xx}}\right)$ after bifurcation. Bifurcation of the growth curve is a qualitative change in the growth dynamic. During bifurcation, two new growth trajectories have been initiated. On the growth trajectory $\left(\mathrm{M}_{\mathrm{x}} \rightarrow \mathrm{M}_{\mathrm{xx}}\right)$, after bifurcation, one set with three species maximum weight phenotypes was identified: $\left.\{A \mid A A ; A a ; a a\}\right|_{M=M x x}$ that correspond to $\left\{A \mid M_{x x 1} ; M_{x x 2} ; M_{x x 3}\right\}$. This set with species maximum weight phenotypes was identified explicitly. It was possible due to the fact that these are asymptotic phenotypes; for the phenotypes ontogenetic growth stopped and no variation at the point is possible. Ontogenetic growth from $\mathrm{m}_{\mathrm{o}}$ up to $\mathrm{M}_{\mathrm{xx}}$ in terms of phenotype ontogenetic transformation is reasonably depict as follows $\mathrm{B} \rightarrow \mathrm{U} \cdot \mathrm{G} \rightarrow \mathrm{A}$.

\section{Conclusions}

○ In the model, the growth phenotypes emerge as a result of an interaction between the feed conversion dynamic and constraints imposed by the weight invariant. The interaction between the processes behind the variables result both in the phenotype-dependant growth curve and the species-specific weight.

$\circ$ The model does not support the opinion that the same genotype can give rise to many different growth phenotypes. Since growing pigs with the same maximum growth rate phenotype can have their individual maximum growth rates this premise is extraneous.

○ The study has identified three sets with the following growth phenotypes:

1. Rapid growth rate phenotypes:

$\{\mathrm{B} \mid \mathrm{BB}, \mathrm{Bb}, \mathrm{bb}\}$ that correspond to $\{\mathrm{B} \mid \mathrm{K}=1,686 ; \mathrm{K}=1,581 ; \mathrm{K}=1,232\}$, respectively;

2. Species maximum weight phenotypes:

$\{\mathrm{A} \mid \mathrm{AA}, \mathrm{Aa}$, aa $\}$ that correspond to $\left\{\mathrm{A} \mid \mathrm{M}_{\mathrm{xx} 1} ; \mathrm{M}_{\mathrm{xx} 2} ; \mathrm{M}_{\mathrm{xx} 3}\right\}$, respectively;

3. Maximum weight phenotypes: $\left\{\mathrm{U} \mid \mathrm{M}=\mathrm{M}_{\mathrm{x}} ; \mathrm{M} \leq \mathrm{M}_{\mathrm{x}} ; \mathrm{M}<\mathrm{M}_{\mathrm{x}}\right\}$.

$\circ$ The sets of growth phenotype are considered as a dynamic system that develops in the course of ontogenetic growth: $\mathrm{B} \rightarrow \mathrm{U} \circ \mathrm{G} \rightarrow \mathrm{A}$.

$\circ$ Rapid growth phenotypes $\{\mathrm{B} \mid \mathrm{BB} ; \mathrm{Bb} ; \mathrm{bb}\}$ can also serve as markers for other quantitative traits that change in accordance with the certain growth phenotype dynamic.

- Conversion of set $\mathrm{B}$ to set $\mathrm{A}$ in natural succession, during ontogenetic growth, is neither smooth nor continuous process.

The processes of growth, it is dynamic of growth phenotype is under genetic control, and the growth trajectories in all stages of growth are phenotype-dependant.

\section{Conflict of interests}

The authors declare that there is no conflict of interests regarding the publication of this paper.

\section{References}

Aihara, K., \& Suzuki, H. (2010). Theory of hybrid dynamical systems and its applications to biological and medical systems. Phil. Trans. R. Soc. A., 368, 4893-4914. 
Altrock, P. M., Liu, L. L., \& Michor, F. (2015). The mathematics of cancer: Integrating quantitative models. Nature Reviews Cancer, 15, 730-745.

Andersson, L., \& Georges, M. (2004). Domestic-animal genomics: deciphering the genetics of complex traits. Nature Reviews Genetics, 5, 202-212.

Cao, H., \& Ibarz, B. (2010). Hybrid discrete-time neural networks. Phil. Trans. R. Soc. A., 368, 5071-5086.

Cobb, J. N., DeClerck, G., Greenberg, C., \& McCouch, S. (2013). Next-generation phenotyping: requirements and strategies for enhancing our understanding of genotype-phenotype relationships and its relevance to crop improvement. Theor. Appl. Genet., 126, 867-887.

Economo, E. P., Kerkhoff, A. J., \& Enquist, B. J. (2005). Allometric growth, life-history invariants and population energetic. Ecology Letters, 8, 353-360.

Fusco, G., \& Minelli, A. (2010). Phenotypic plasticity in development and evolution: facts and concepts. Phil. Trans. R. Soc. B., 365, 547-556.

Hill, W. G. (2010). Understanding and using quantitative genetic variation. Philosophical Transactions of the Royal Society B, 365, 73-85.

Hou, C., Bolt, K. M., \& Bergman, A. (2011). A general model for ontogenetic growth under food restriction. Proc $R$ Soc B., 278, 2881-2890.

Houle, D. (2010). Numbering the hairs on our heads: The shared challenge and promise of phenomics. PNAS, 107(1), 1793-1799.

Houle, D., Govindaraju, D., \& Omholt, S. (2010). Phenomics: The next challenge. Nature Reviews Genetics, 11, 855-866.

Jaeger, J., \& Monk. N. (2014). Bioattractors: Dynamical systems theory and the evolution of regulatory processes. J. Physiol., 592(11), 2267-2281.

Li, Y., \& Wu, R. (2010). Functional mapping of growth and development. Biol. Rev., 85, 207-216.

Magowan, E., McCann, M. E. E., Beattie, V. E., McCracken, K. J., .. \& Mayne, C. S. (2007). Investigation of growth rate variation between commercial pig herds. Animal, 1(8), 1219-1226.

Mailleret, L., \& Lemesle, V. (2009). A note on semi-discrete modelling in the life sciences. Phil. Trans. R. Soc. A., 367, 4779-4799.

Marjoram, P., Zubair, A., \& Nuzhdin, S. V. (2013). Post-GWAS: Where next? More samples, more SNPs or more biology? Heredity, 112, 79-88.

Nijhout, H. F., \& German, R. Z. (2012). Developmental Causes of Allometry: New Models and Implications for Phenotypic Plasticity and Evolution. Integrative and Comparative Biology, 52(1), 43-52.

Nijhout, H. F., Roff, D. A., \& Davidowitz, G. (2010). Conflicting processes in the evolution of body size and development time. Phil.Trans. R. Soc. B., 365, 567-575.

Noble, D. (2008). Genes and causation. Phil. Trans. R. Soc. A., 366, 3001-3015.

Trudy, F. C., Mackay, E., Stone, A., \& Ayroles, J. F. (2009). The genetics of quantitative traits: Challenges and prospects. Nature Reviews Genetics, 10, 565-577.

Werner, J., \& Griebeler, E. M. (2011). Reproductive Biology and Its Impact on Body Size: Comparative Analysis of Mammalian, Avian and Dinosaurian Reproduction. PLoS ONE, 6(12), e2844.

Wu, R., Ma, C. X., Zhao, W., \& Casella, G. (2003). Functional mapping for quantitative trait loci governing growth rates: A parametric model. Physiol Genomics., 14, 241-249.

\section{Copyrights}

Copyright for this article is retained by the author(s), with first publication rights granted to the journal.

This is an open-access article distributed under the terms and conditions of the Creative Commons Attribution license (http://creativecommons.org/licenses/by/4.0/). 